\title{
Research Paper \\ Determining Cardiometabolic and Antioxidant Effects of Olive Leaf Extract in Patients with Essential Hypertension
}

\section{Hayedeh Yaghoobzadeh ${ }^{1} \mathrm{C}$, Sepideh Mehravar ${ }^{1}$, Hamidreza Javadi²,3, Mohammad Reza Memarzadeh ${ }^{4}$, ${ }^{*}$ Seyyed Mehdi Mirhashemi ${ }^{2} \mathrm{C}$}

1. Student Research Committee, Qazvin University of Medical Sciences, Qazvin, Iran.

2. Metabolic Diseases Research Center, Research Institute for Prevention of Non-Communicable Diseases, Qazvin University of Medical Sciences, Qazvin, Iran.

3. Department of Cardiology, School of Medicine, Qazvin University of Medical Sciences, Qazvin, Iran.

4. Medicinal Plants Research Center of Barij, Kashan, Iran.

\begin{tabular}{|c|c|}
\hline $\begin{array}{l}\text { Use your device to scan } \\
\text { and read the article online }\end{array}$ & Citation Yaghoobzadeh H, Mehravar S, Javadi HR, Memarzadeh MR, Mirhashemi SM. Determining Cardiometabolic and Antioxi- \\
\hline atsing & $\begin{array}{l}\text { dant Effects of Olive Leaf Extract in Patients with Essential Hypertensio. The Journal of Qazvin University of Medical Sciences. 2019; } \\
\text { 23(5):372-381. https://doi.org/10.32598/JQUMS.23.5.372 }\end{array}$ \\
\hline abtsta & d of https://doi.org/10.32598/JQUMS.23.5.372 \\
\hline
\end{tabular}

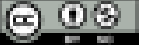

Received: 07 Aug 2019 Accepted: 12 Oct 2019 Available Online: 01 Dec 2019

Keywords: Lipid profiles, Blood pressure, Malondialdehyde, Superoxide dismutase, Olive leaf extract

\section{A B STR ACT}

Background Hypertension is one of the most common clinical disorders affecting millions worldwide. Some studies have indicated the role of oxidative stress in the pathogenesis of hypertension and the importance of antioxidant compounds in their control.

Objective The aim of this study was to evaluate the effects of olive leaf extract (OLE), Olea europaea L., on cardiometabolic parameters and biomarkers of oxidative stress in patients with essential hypertension. Methods This randomized double-blind placebo-controlled clinical trial conducted in 2017 on 60 patients with essential hypertension aged 30-60 years referred to the cardiovascular clinic of Bu-Ali Sina Hospital in Qazvin, Iran. The patients were randomly allocated into two groups of OLE ( $n=30$; receiving OLE 250 mg capsules twice per day for 12 weeks) and placebo ( $n=30$, receiving placebo drug for 12 weeks). Before and after intervention, cardiometabolic parameters and oxidative stress biomarkers were measured using appropriate laboratory methods. To compare variables and groups, paired-t test and independent t-test were used, respectively.

Findings The OLE intake led to a significant decrease in systolic blood pressure, serum total cholesterol, and malondialdehyde levels in hypertensive patients compared to the placebo group, but significantly increased superoxide dismutase activity $(P<0.05)$. Moreover, the OLE intake had no significant effect on diastolic blood pressure, other lipid profiles and biomarkers of oxidative stress ( $P>0.05)$.

Conclusion OLE intake for 12 weeks had beneficial effects on some types of cardiometabolic and oxidative stress biomarkers in hypertensive patients.

\section{Extended Abstract}

\section{Introduction}

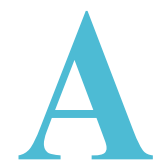

$\mathrm{n}$ individual with systolic blood pressure (SBP) above $140 \mathrm{mmHg}$ or diastolic blood pressure (DBP) above 90 $\mathrm{mmHg}$ is considered as a hypertensive patient [1]. Many factors such as genet- ics, family history, environmental factors and social and behavioral characteristics have critical role in the incidence of hypertension [2]. Despite our understanding of the disease and accessibility of treatments, it was estimated that around $30 \%$ of the world's population will be affected by this disease by 2025 , indicating a major unrelieved public health burden [3-5]. Herbal medicine has been used for a long time to achieve therapeutic goals [6]. Extracts of olive leaves (Olea

* Corresponding Author:

Seyyed Mehdi Mirhashemi

Address: Metabolic Diseases Research Center, Research Institute for Prevention of Non-Communicable Diseases, Qazvin University of Medical Sciences, Qazvin, Iran.

Tel: +98 (28) 3336001

E-Mail: mirhashemism@gmail.com 
europaea L.) are abundant in polyphenols especially oleuropein. Cardio protective, antioxidant and anti-inflammatory properties of Olive Leave Extracts (OLEs) have been demonstrated in many studies [7, 8]. The positive effects of Olea europaea L. in inducting apoptosis and antioxidant properties in human colon and cervical cancer cell lines have already been reported $[9,10]$. In another study in 2019, improvement of lipid and antioxidants levels following treatments by 400 $\mathrm{mg} / \mathrm{kg}$ OLE was reported in streptozotocin induced diabetic rats [11].

De Bock et al. stated that the use of these extracts has no effect on blood pressure and plasma lipids in male subjects with overweight [12], but in contrary, Filip et al. showed that the use of OLE can reduce SBP and DBP in persons with hypertension. They also reported its positive effect on lipid profiles improvement in subjects with normal level of blood lipid and/or hypercholesterolemia [13]. Since clinical trials on the effects of OLEs are very limited [14], in order to have better understanding of the impact of OLEs and al to address the contradictory results, this randomized, controlled, double-blind trial aimed to examine the effect of OLE on cardiometabolic parameters of blood pressure and lipid profile and oxidative stress biomarkers in patients with hypertension.

\section{Materials and Methods}

This study is a randomized double-blind placebocontrolled clinical trial conducted on 60 patients with essential hypertension aged 30-60 years referred to the cardiovascular clinic of Bu-Ali Sina Hospital affiliated to Qazvin University of Medical Sciences in Iran. Participants were assigned into two matched groups of OLE (receiving 250-mg OLE twice per day for 12 weeks) and placebo. Placebo and OLE capsules were prepared in Barij Medicinal Plants Research Center in Kashan, Iran.

Study variables were cardiometabolic parameters (SBP and DBP and serum lipids) and oxidative stress biomarkers. The blood pressure of the participants was measured by using a standard mercury sphygmomanometer. Serum lipid levels were quantified by BT-1500 autoanalyzer (Biotechnica, Italy) and DiaLab kit. Oxidative stress biomarkers including total antioxidant capacity (TAC) [18], glutathione (GSH) [19], Malondialdehyde (MDA) [20] were measured using a spectrophotometric method. To evaluate superoxide dismutase (SOD) activity, colorimetric method based on the reaction of nitro-blue tetrazolium chloride with superoxide radicals and formation of a blue formazan [21]. To compare variables before and after treatment and for between-group comparison, paired-samples and independent t-test were used, respectively. Results were presented as Mean \pm SD and the significance level was set at $\mathrm{P} \leq 0.05$.

\section{Results}

Sixty patients were participated in this study. Relatively, there was high level of compliance in this study such that more than $90 \%$ of OLE capsules were used by the patients. No complication was reported follow-

Table 1. Characteristics of the study subjects $(n=30)$

\begin{tabular}{cccc}
\hline & \multicolumn{2}{c}{ Means \pm SD/ No. (\%) } & P \\
\cline { 2 - 4 } Variables & OLE Group & Placebo Group & 0.972 \\
\hline Gender & 15 males/ 15 females & 15 males/ 15 females & 0.114 \\
\hline Age (Year) & $52.9 \pm 10.3$ & $57.9 \pm 10.8$ & 0.802 \\
\hline Height (cm) & $159.2 \pm 9.1$ & $158.5 \pm 9.3$ & 0.364 \\
\hline Weight (kg) & $74.1 \pm 9.1$ & $71.6 \pm 11.1$ & 0.779 \\
\hline ACEI/ARB drugs & $21(70)$ & $22(73)$ & 0.799 \\
\hline$\beta$-blockers & $13(43.3)$ & $14(46.7)$ & 0.612 \\
\hline Calcium channel blockers & $17(56.7)$ & $15(53.3)$ & $>0.999$ \\
\hline
\end{tabular}


ing its consumption among hypertensive patients. The two groups were matched for sex, age, and height, type and dosage of drugs and hence, no significant differences were found between them $(\mathrm{P}>0.05)$ (Table 1).

After intervention, OLE intake led to a significant reduction in SBP $(\mathrm{P}=0.026)$, total cholesterol $(\mathrm{P}=0.029)$, and MDA ( $\mathrm{P}=0.013)$ as well as a significant increase in high-density lipoprotein cholesterol $(\mathrm{P}=0.041)$ and SOD activity $(\mathrm{P}<0.001)$ compared to the placebo group. OLE intake had no statistically significant effect on other measured cardiometabolic parameters and oxidative stress biomarkers $(\mathrm{P}>0.05)$.

\section{Discussion}

Consistent with our study, Susalit et al. reported that using $1000 \mathrm{mg} / \mathrm{d}$ OLE for eight weeks resulted in small reduction in clinic SBP and total cholesterol compared to baseline values, but they demonstrated that this dose of OLE can lead to reduction in triglyceride and lowdensity lipoprotein cholesterol levels which is against our results [15]. The most important advantage of our study compared to their study was the presence of a placebo group. In a randomized, double-blind, controlled, crossover study conducted by Lockyer et al., it was shown that the OLE usage increases hypotensive and lipid-lowering effects in vivo [24].

Khalili et al. showed that $60 \mathrm{mg} / \mathrm{kg}$ /day Oleuropin significantly reduced the blood pressure, total cholesterol, low-density lipoprotein cholesterol, triglyceride, and MDA levels. It also increased serum high-density lipoprotein and erythrocyte SOD activity in rats with simultaneous renovascular hypertension and type 2 diabetes [27]. A possible reason that OLE intake cause antihypertensive and hypolipemic effects may be their association with cellular redox modulatinon effects [29]. OLE is an important source of natural antioxidants; it has effective antioxidant activity against different reactive species and protects human erythrocytes against oxidative damage [30]. The most important difference between our study and the previous studies may be related to the study design, type of disease, nature of the extract, duration of the extract intake, patients' compliance and the inclusion/exclusion criteria. This study had some limitations. Evaluation of gene expressions related to metabolism, inflammation and oxidative stress after intervention in hypertensive patients are recommended for further studies.

Overall, it was concluded that, in hypertensive patients, OLE administration had beneficial effects on
SBP, total cholesterol, high-density lipoprotein cholesterol, MDA levels and SOD activity, but had no effect on other cardiometabolic parameters.

\section{Ethical Considerations}

\section{Compliance with ethical guidelines}

This study was approved by the Research Ethics Committee of Qazvin University of Medical Sciences (Code: IR.QUMS.REC.1395.264) and is a registered clinical trial (Code: IRCT2017043033730N1).

\section{Funding}

This work was financially supported by the Deputy for Research and Technology of the Qazvin University of Medical Sciences.

\section{Authors' contributions}

Conceptualization: Seyyed Mehdi Mirhashemi; Investigation and data analysis: Seyyed Mehdi Mirhashemi, Hayedeh Yaghoobzadeh, Sepideh Mehravar, and Hamidreza Javadi; Preparation of the medications: Mohammad Reza Memarzadeh; Draft preparation: Seyyed Mehdi Mirhashemi and Hayedeh Yaghoobzadeh.

\section{Conflicts of interest}

The authors declare no conflict of interest. 


\title{
تعيين اثرات كارديو متابوليك و آنتىاكسيدانى عصاره برتى زيتون در بيمار ان هايير تانسيون اوليه
}

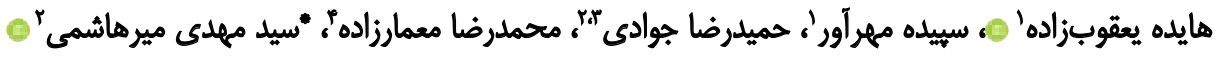 \\ 1. كميته تحقيقات دانشجويى دانشكاه علوميزشكى قزوين، قزوين، ايران.

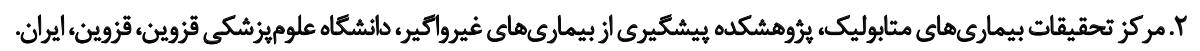

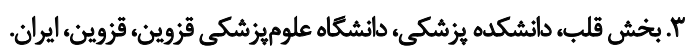 \\ F. مركز تحقيقات تياهان دارويى باريج، كاشان، ايران.
}

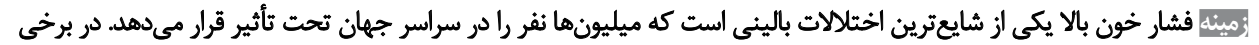

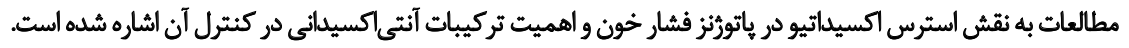

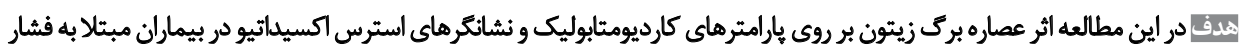
خون اوليه بررسى شد.

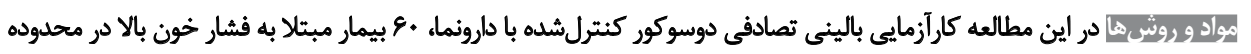

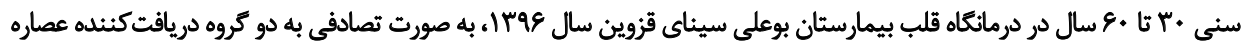

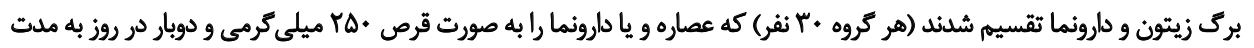

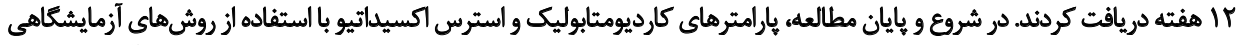

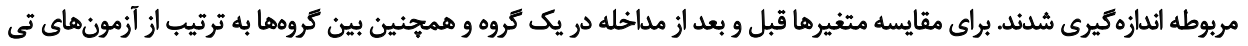
زوجى وتى مستقل استفاده شد.

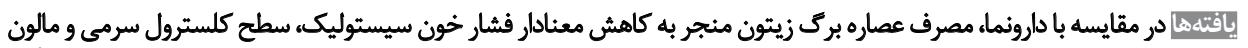

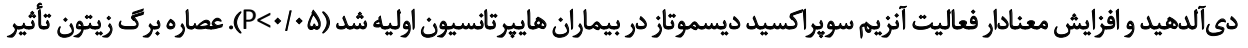

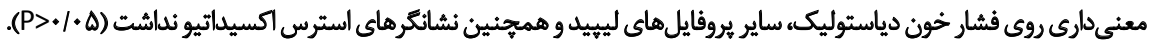

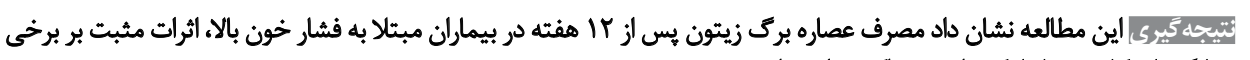
نشانكرهاى كارديومتابوليك واسترس اكسيداتيو داشت.
\end{abstract}

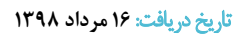

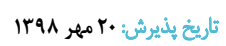

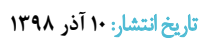

كليدوأوهاء:

يروفايلهاي ليبيد، فشار

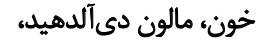
سوير اكسيد ديسموناز، عصاره بركى زيتون

ماروهاى كياهى به مدت طولانى براى دستيابى به اهداف درمانى

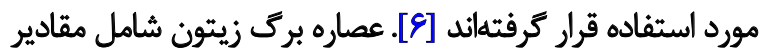

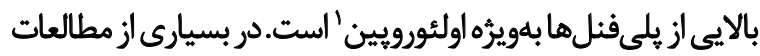

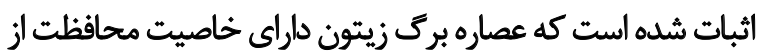

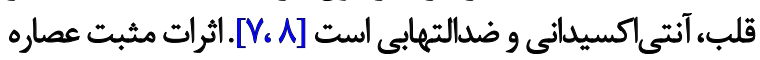

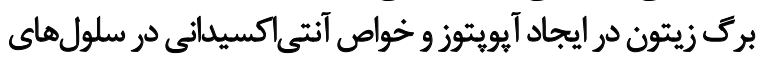

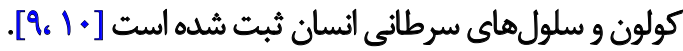

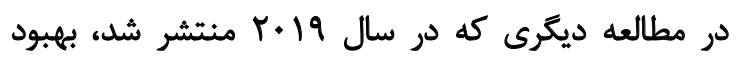

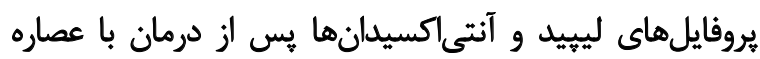

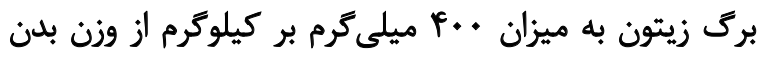

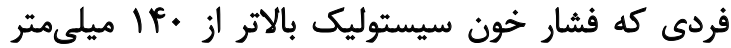

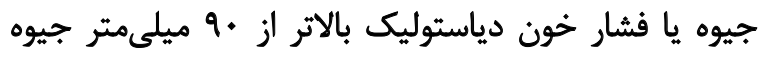

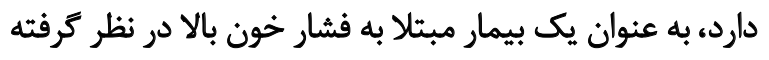

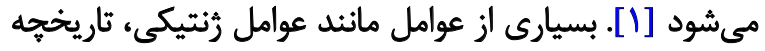

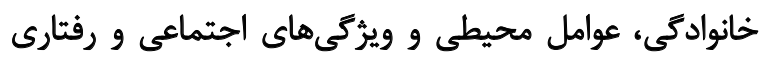

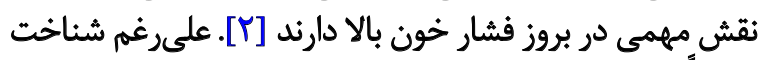

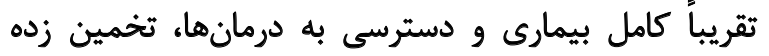

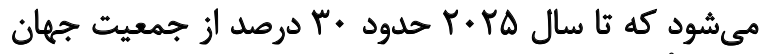

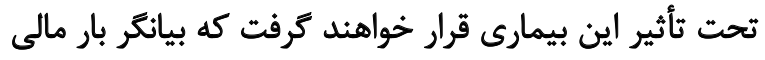

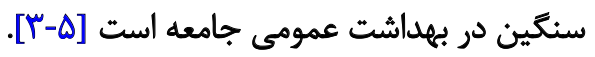


نشانكر هاي استرس اكسيداتيو، به عنوان غيامد مورد ارزيابى قرار

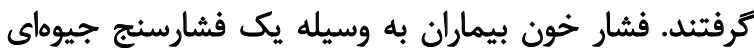

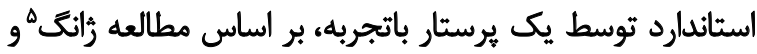

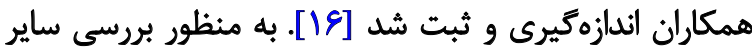

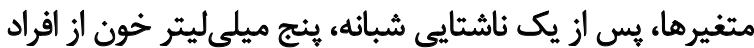

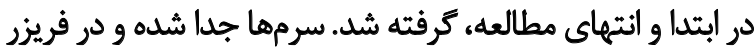

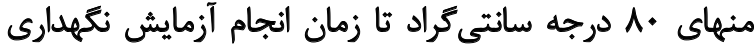

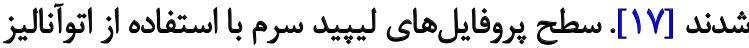

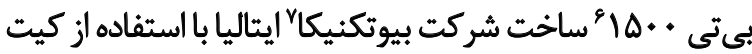

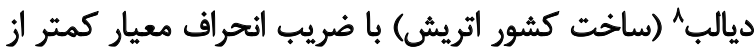

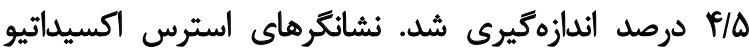

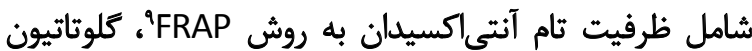

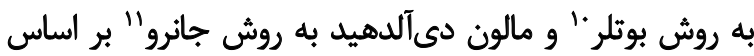

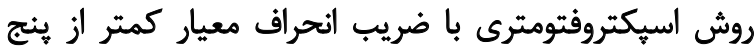

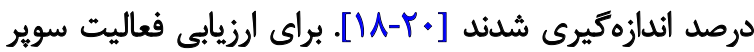

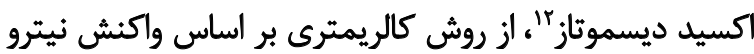

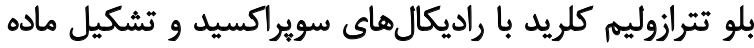

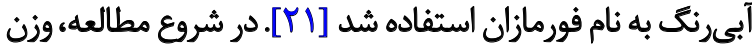

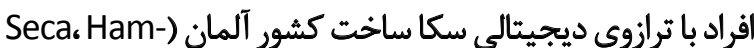
اندازمكيرى شد و اطلاعات داروها از طريق المبان (burg، Germany

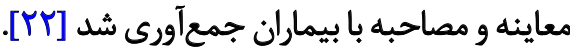
ازنرمافزار SPSS،نسخه بr جهت تجزيه و تحليل دادهها استفاده

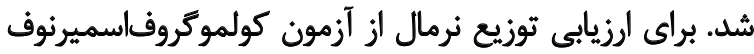

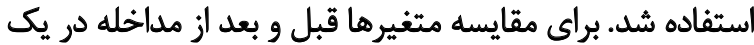

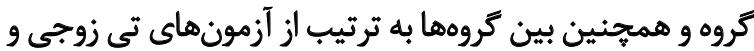

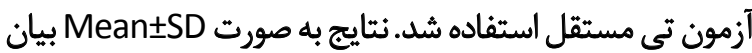
شدند و سطح معنادارى، كمتر از هـ ٪ • در نظر كرفته شد.

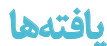

در ابتدا •V Vفر بيمار مبتلا به فشار خون مورد ارزيابى قرار

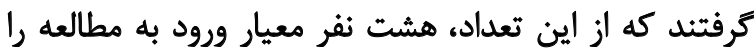

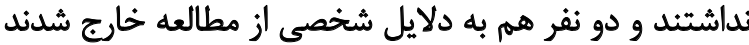

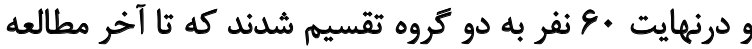

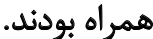
سطح انطباق در اين مطالعه بالا بود؛ به طورى كه بيش

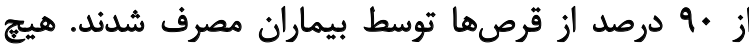
عارضه ناخواستهاى در بيماران مصرف كنينده عصاره ديده نشد.

9. Ferric reducing ability of plasm (FRAP)

10. Beutler

11. Janero

12. Superoxide dismutase (SOD)
در موشهاى صحرايى ديابت شده با استريتوزوتوسين تأييد شد إندان

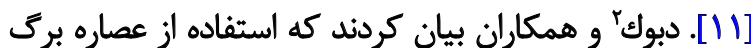

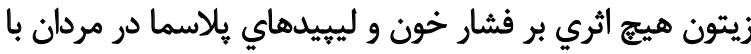

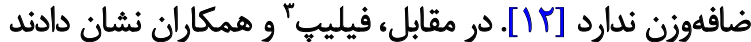

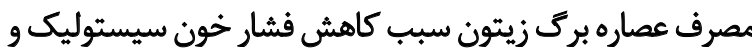

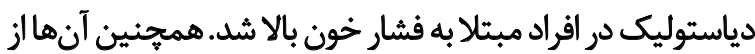

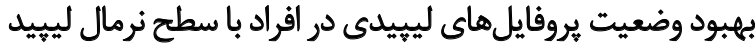

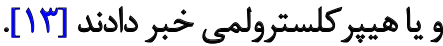
از آنجا كه مطالعات كارآزمايى بالينى انجامشده در مورد اثرات

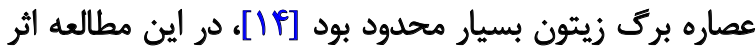

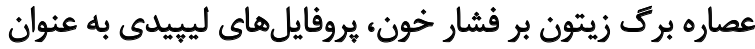

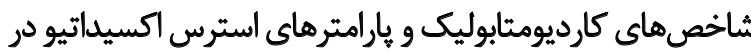
بيماران مبتلا به فشار خون بالا ارزيابى شدائ بارئ

\section{مواد ورشها نها}

اين يُروهش، يك كارآزمايى بالينى تصادفى دوسوكور

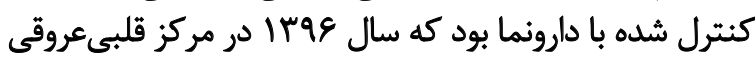

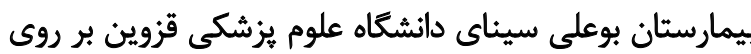

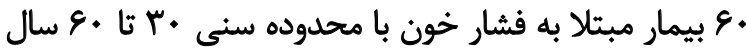

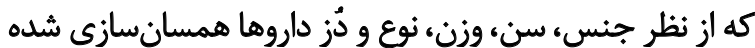

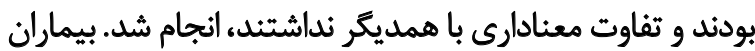

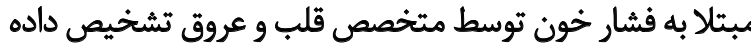

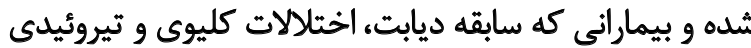

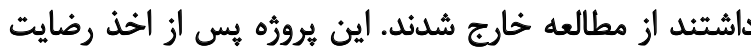

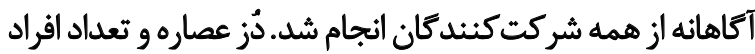

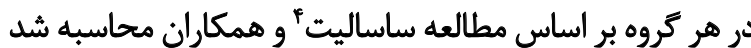

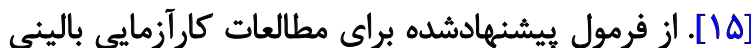
جهت تعيين تعداد افراد استفاده شد.

افرادباستفاده از نرمافزار اعداد تصادفى به دو كروه دريافت كنينده

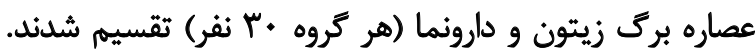

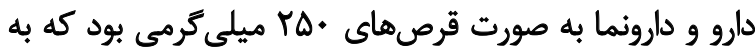

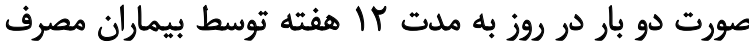

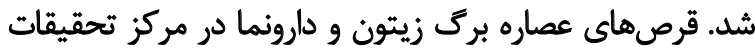

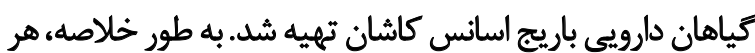

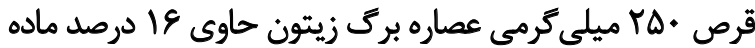

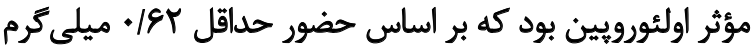

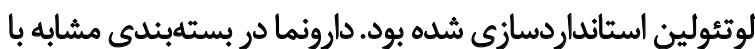
عصاره و حاوى ميكروكريستالين سلولز بود. در بيمار ان، فشار خون سيستوليك، دياستوليك و بروفايلهاى ليبيدى به عنوان شاخصهاي كارديون مثابوليك و هميجين

4. Susalit 
مصرف هزار ميلى كرم عصاره برك زيتون در روز در طى هشت

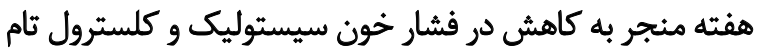

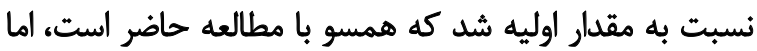

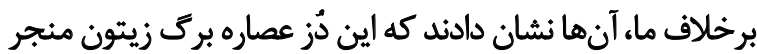

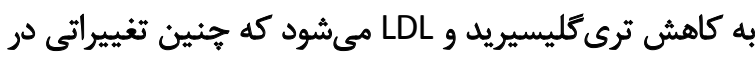

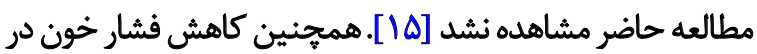

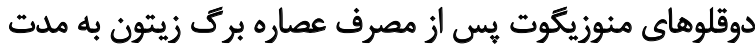

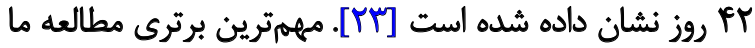

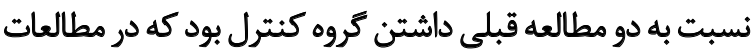

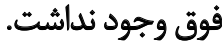

در يك كارآزمايى تصادفى دوسوكور كنترلشده مثقاطع كه

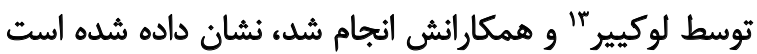

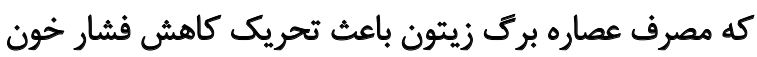

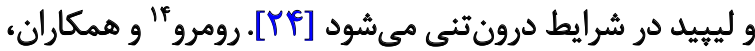

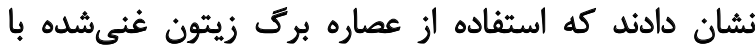

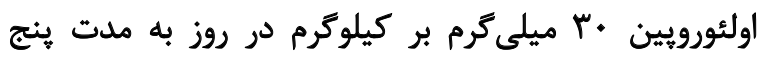

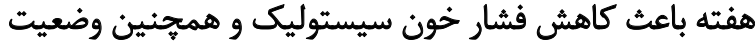

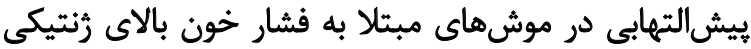

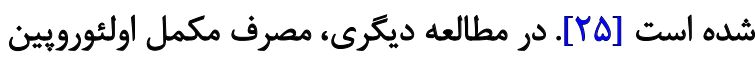

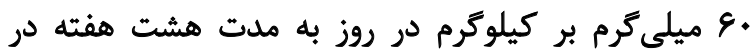

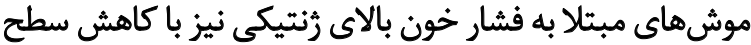

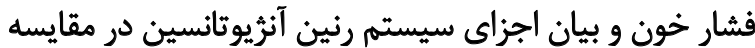

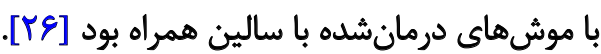

خليلى و همكاران نشان دادند كه اولئورويَين •و ميلى كرم بر كيلوكرم در روز به طور قابل توجهي سبب كاهش فان فشار خون،

13. Lockyer

14. Romero
دو گروهاز نظر جنس، سن، وزن، نوع و دُز داروها همسانسازى (هان

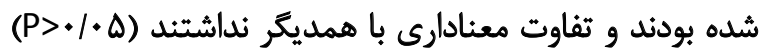

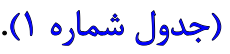

در مقايسه با كروه دارونما، مصرف عصاره برك زيتون سبب

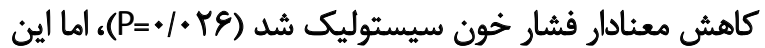

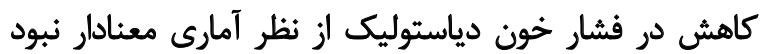

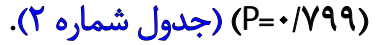

به منظور ارزيابى اثر عصاره برى زيتون بر يروفايل هاي ليتيديدي،

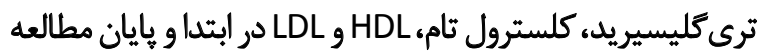

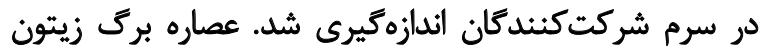

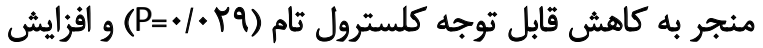

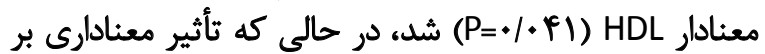

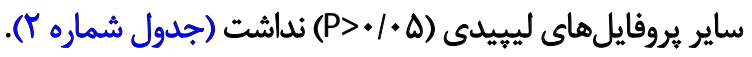
با با IT أهته مصرف عصاره برك زيتون، كاهش معنادار سطح

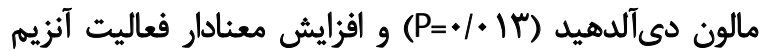

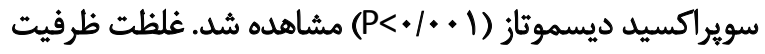

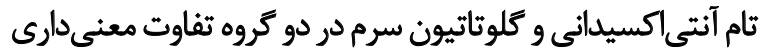

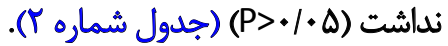

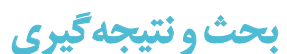

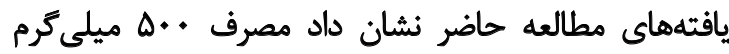

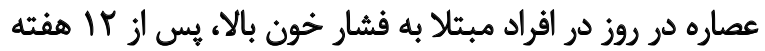

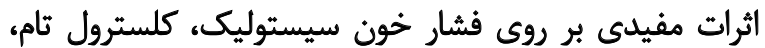

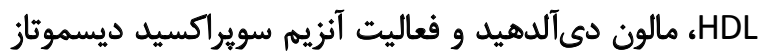

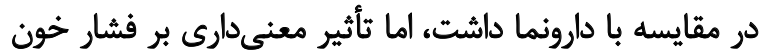

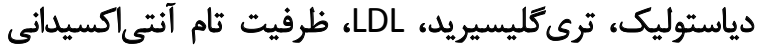
و كلوتاثيون نداشت. ساساليت و همكاران، كزارش دارئ دادند كه

جدول (ـ مشخصات عمومي افراد شركت كنيده در مطالعه (در هر كروه · ب نقر) با استفاده از آزمون تي مستقل

\begin{tabular}{|c|c|c|c|}
\hline \multirow{2}{*}{ سطح معنى دارى } & \multicolumn{2}{|c|}{ مياثكين土|نحراف معيار / تعداد (درصد) } & \multirow{2}{*}{ متغيرها } \\
\hline & تمروه دريافت كننده دارونما & تروه دريافت كنثده عصاره برك زيتون & \\
\hline.$/ 94 \pi$ & $1 \Delta / 1 \Delta$ & $1 \Delta / 1 \Delta$ & جنس (مرد/زن) \\
\hline.$M F$ & $\Delta V / q \pm 1 \cdot / \Lambda$ & $\Delta r / q \pm 1 \cdot / r$ & سن (سال) \\
\hline$+/ A \cdot r$ & $1 \Delta N \Delta \pm q / \Gamma$ & $|\Delta Q / T \pm V|$ & قلد (سائتى مثت) \\
\hline$+/$ Wat & $V / / \& \pm 1 / / 1$ & $n+/ \pm \pm q / 1$ & وزن (كيلوكرم) \\
\hline.$/ W 9$ & $M(M)$ & $M\left(V_{+}\right)$ & 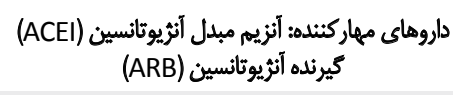 \\
\hline.$/ 299$ & if $(r / M)$ & $\mathbb{I T}(\Re / M)$ & داروهاي مهاركنئه بتا \\
\hline +/air & $1 \Delta(\Delta H / T)$ & $\operatorname{IV}(\Delta s / V)$ & مسلودكنثدهاى كانال كلسيم \\
\hline$>. / 999$ & $\Delta(1 / N)$ & $\Delta(1 \& / N \Delta)$ & داروهاى تركيبى \\
\hline
\end{tabular}


جدول r. اثر عصاره برك زيتون و دارونما بر روى فشار خونء بروفايل هاى ليجيدى و نشانكرهاى

\begin{tabular}{|c|c|c|c|c|c|c|c|c|c|c|}
\hline \multirow{3}{*}{ 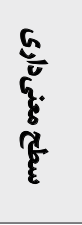 } & \multicolumn{8}{|c|}{ ميانكين+انحراف معيار } & \multirow{3}{*}{\multicolumn{2}{|c|}{ متغيرها }} \\
\hline & \multicolumn{4}{|c|}{ كروه دريافت كنثده دارونما } & \multicolumn{4}{|c|}{ تروه دريافت كثنده عصاره بريى زيتون } & & \\
\hline & معنى سطحي & تغييرات & اتتهيا & ابتدا & معنى سطحى & تغييرات & اتثها & ابتدا & & \\
\hline.$/ . r e "$ &.$/ M T$ & $-r / I \pm 1 \cdot / f$ & $|r N| \pm V / K$ & $I f \cdot / N \pm V / q$ & $.1 . .1$ & $-\sqrt{ } \pm \pm \sqrt{ } g$ & $|r| F \mid E V / F$ & $|\mp+/| \pm N \Delta$ & فشارخون سيستوليك' & c. \\
\hline.$/ 199$ &.$/ W$ & $-r / T \pm \sqrt{ } / \lambda$ & $\Lambda e / N \pm V / \varphi$ & $A V / / \pm V / q$ & $.1 .9 \%$ & $-r \pm 1 V / r$ & $N \mid \mathscr{E} \pm N \Delta$ & $9 . / \% \pm N H$ & فشار خون دياستوليك، & \\
\hline$+|\Delta| \mid$ & $+M F T$ & $-r \pm \Pi r / q$ & $W F / N \pm T / F$ & $W W \Psi T Y / \Delta$ & (THD & $-g / \Delta \pm r v r$ & $\mid N F / \Delta \pm r V / q$ & $19 \cdot / q \pm r V / V$ & (ميلى كرم بردسيىيتر) & \\
\hline.$/ .99^{\circ}$ &.$/$ TA & $-Y / \& \pm M / T$ & MVA $\pm I V E$ & $m / 1 \pm \pi / 9$ & $.1 . .1$ & $-1 Q / T \pm Y V / Y$ & $T / W / \Delta \pm Y V / \Delta$ & $T N E \pm T / K$ & (ميلى كرمبردستىليت) & $\frac{G}{E}$ \\
\hline.$+\% 1^{*}$ & $+/ P T$ & $+/ \mid \backslash \pm \Delta / 1$ & $F A / t \pm F / \Delta$ & $\mu \neq / v \pm \Psi / \mu$ & $t+t+r$ & $r / \lambda \pm F / \&$ & $E / / \pm E / F$ & $\Gamma / \Psi \pm \Delta / \gamma$ & 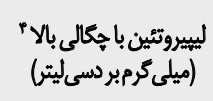 & $\bar{\xi}$ \\
\hline$\cdot / \Lambda \cdot \Delta$ & DFT & $-F / \backslash \pm \mid a / g$ & $\mid Y N Y \pm 1 . / 9$ & $\mid \pi / \pi \pm 1 \cdot / f$ &.$/ \pi n$ & $-r / q \pm 1 \cdot / A$ & $\mid r / T \pm 1 . / T$ & $I T / T \pm I V / r$ & 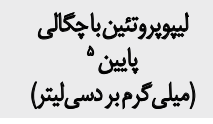 & \\
\hline 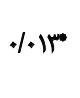 & $+/ 199$ & $-+/ T E \pm / \Delta Y$ & $\Delta / / q \pm \cdot / T$ & $\Delta / r q \pm+/ F q$ & $+1++1$ & $-* / Y \pm \pm / M q$ & $F / F \Delta \pm \cdot / F q$ & $\Delta / / \& \pm+/ \Delta Y$ & (ميلى كرمبردسى ألدهيد) & \\
\hline$+/ F \mathrm{AM}$ & HAPY & $\| W / I T \pm N \Lambda$ & $\Lambda F Y / \Lambda \pm 9 Y / /$ & $\Lambda \Pi \sim / \perp \pm N / V$ & $+/ m n^{\circ}$ & rVI $\pm 1 \% / 1$ & $N \Delta N \backslash \pm 9 * / 9$ & $A T N \backsim \pm N \Delta$ & 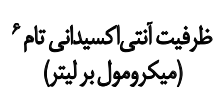 & \\
\hline.$\cdot .1^{*}$ &.$/ F A \Delta$ & $-.|\beta| \pm \Psi / f$ & $\mid \Psi / \Psi \pm \Psi / \Delta$ & $\mid r / q \pm 1 / r$ & $.1 . .1$ & $r / \Delta \pm t / \lambda$ & WQET/G & $\mid f / x \pm \pi / f$ & 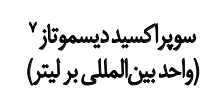 & $\underline{\xi}$ \\
\hline / TaY & $+/ Y A Y$ & $-r / q \pm V V \Lambda$ & $M I S / N \pm F V / q$ & $\pi \cdot / \Gamma \pm \mathscr{R} T / T^{\circ}$ & $+M$ & $I V / V \pm W / 4$ & 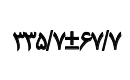 & $M M \pm N A / Y$ & (ميكرومول برليثر) & \\
\hline
\end{tabular}

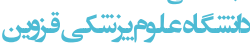

1. Systolic blood pressure

2. Diastolic blood pressure

3. Triglyceride

4. High density lipoprotein

كاهشى بر روى فشار خون و بروفايلهاى ليجيد مى شوداد، ممكن

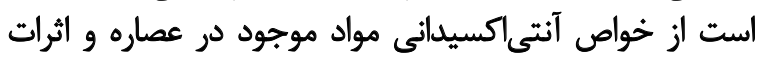

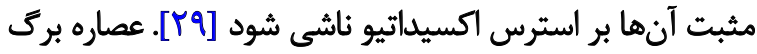

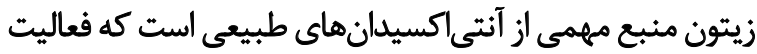

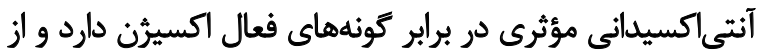

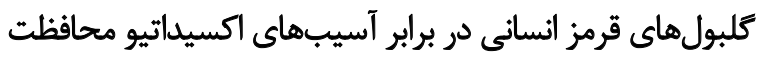

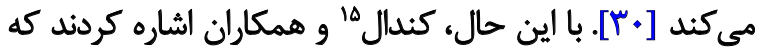

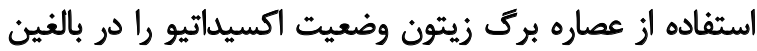

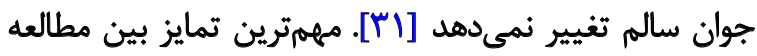

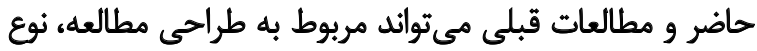

• سطح معنى دارى

5. Low density lipoprotein

6. Total antioxidant capacity

7. Superoxide dismutase

كلسترول تام، ترى كليسيريد، LDL، مالون دى آلدهيد و افزايش

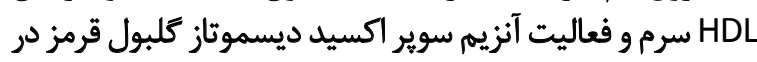

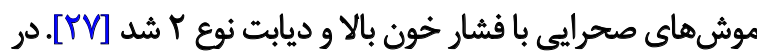

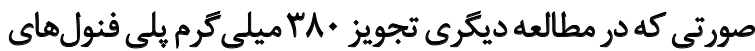

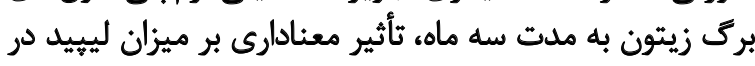

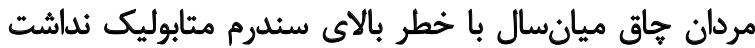

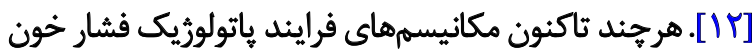

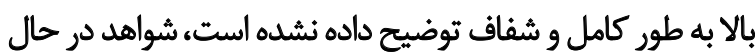

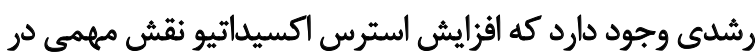

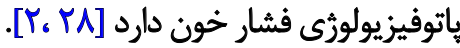
مكانيسم احتمالى كه عصاره برك زيتون سبب ايجاد اثرات 
بيمارى، ماهيت عصاره، مدت مصرف عصاره، رضايت بيمار و و بردي

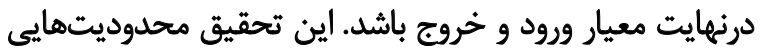

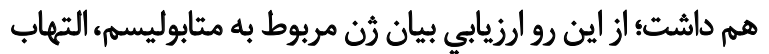

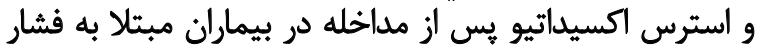
خون بالادر مطالعات آينده توصيه مىشود.

مطالعه حاضر يكى از محدود مطالعاتى است كه اثرات عصاره

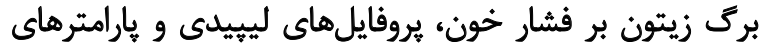

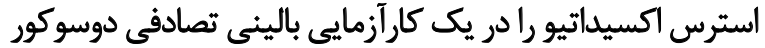

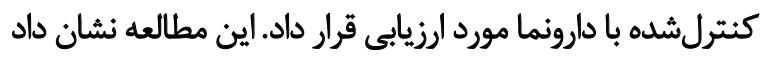

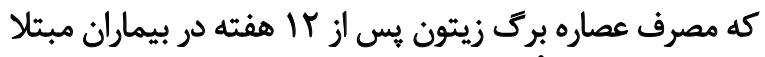

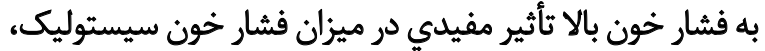

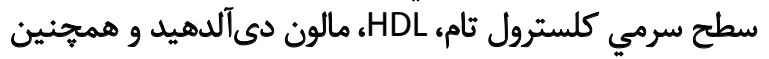

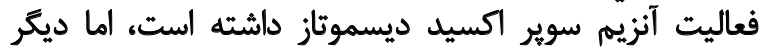
نشانكرهاى كارديومتابوليك و استرس اكسيداتيو راتئيت ديير نداد.

مالامثات أخلاق

\section{بيروى از اصول اخلاق يُؤهش}

اين مطالعه توسط كميته اخلاق دانشعاه علوم يزشكى قزوين

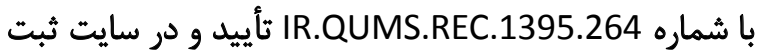
كارآزمايى بالينى ايران با شماره IRCT2017043033730N1 تايد ديت دايت

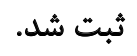

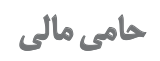

اين مقاله با حمايت مالى معاونت تحقيقات و فناورى دانشكاه

علوم يزشكى قزوين انجام شده است.

$$
\text { مشاركت نويسندكان }
$$

طراح: سيد مهلدى ميرهاشمى؛ جمع آورى دادها، تحليل و تفسير

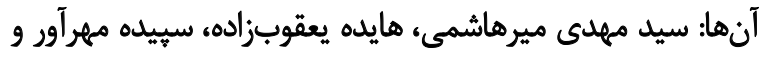

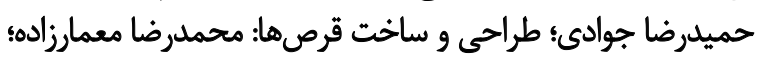

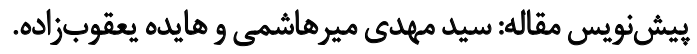

$$
\text { ت تعارض مثاقع }
$$

بنابر اظهار نويسندكان، اين مقاله تعارض منافع ندارد. 


\section{References}

[1] Mozaffarian D, Benjamin EJ, Go AS, Arnett DK, Blaha MJ, Cushman M, et al. Heart disease and stroke statistics-2016 update: A report from the American Heart Association. Circulation. 2016; 133(4):e338-60.

[2] Ahmad KA, Yuan Yuan D, Nawaz W, Ze H, Zhuo CX, Talal B, et al. Antioxidant therapy for management of oxidative stress induced hypertension. Free Radic Res. 2017; 51(4):428-38. [DOI:1 0.1080/10715762.2017.1322205] [PMID]

[3] Nwankwo T, Yoon SS, Burt V, Gu Q. Hypertension among adults in the United States: National Health and Nutrition Examination Survey, 2011-2012. NCHS Data Brief. 2013; (133):1-8.

[4] Sinha N, Kumar Dabla P. Oxidative stress and antioxidants in hypertension-a current review. Curr Hypertens Rev. 2015; 11(2):132-42. [DOI:10.2174/1573402111666150529130922] [PMID].

[5] Wang W, Hu SS, Kong LZ, Gao RL, Zhu ML, Wang WY, et al. Summary of report on cardiovascular diseases in China, 2012. Biomed Environ Sci. 2014; 27(7):552-8.

[6] Guex CG, Reginato FZ, Figueredo KC, da da Silva ARH, Pires FB, da Silva Jesus R, et al. Safety assessment of ethanolic extract of Olea europaea $L$. leaves after acute and subacute administration to Wistar rats. Regul Toxicol Pharmacol. 2018; 95:395-9. [DOI:10.1016/j.yrtph.2018.04.013] [PMID].

[7] Magrone T, Spagnoletta A, Salvatore R, Magrone M, Dentamaro $F$, Russo MA, et al. Olive leaf extracts act as modulators of the human immune response. Endocr Metab Immune Disord Drug Targets. 2018; 18(1):85-93. [DOI:10.2174/18715303176661711 16110537] [PMID].

[8] Sarbishegi M, Gorgich EAC, Khajavi O. Olive leaves extract improved sperm quality and antioxidant status in the testis of rat exposed to rotenone. Nephrourol Mon. 2017; 9(3):e47127. [In Persian] [DOI:10.5812/numonthly.47127].

[9] Hernández-Corroto E, Marina ML, García MC. Multiple protective effect of peptides released from Olea europaea and Prunus persica seeds against oxidative damage and cancer cell proliferation. Food Res Int. 2018; 106:458-67. [DOI:10.1016/j. foodres.2018.01.015] [PMID].

[10] Zeriouh W, Nani A, Belarbi M, Dumont A, de Rosny C, Aboura I, et al. Correction: Phenolic extract from oleaster (Olea europaea var. Sylvestris) leaves reduces colon cancer growth and induces caspase-dependent apoptosis in colon cancer cells via the mitochondrial apoptotic pathway. PloS One. 2017; 12(4):e0176574. [DOI:10.1371/journal.pone.0176574] [PMID] [PMCID].

[11] Al-Attar AM, Alsalmi FA. Effect of Olea europaea leaves extract on streptozotocin induced diabetes in male albino rats. Saudi J Biol Sci. 2019; 26(1):118-28. [DOI:10.1016/j.sjbs.2017.03.002] [PMID] [PMCID].

[12] De Bock M, Derraik JG, Brennan CM, Biggs JB, Morgan PE, Hodgkinson SC, et al. Olive (Olea europaea L.) leaf polyphenols improve insulin sensitivity in middle-aged overweight men: A randomized, placebo-controlled, crossover trial. PloS One. 2013; 8(3):e57622. [DOI:10.1371/journal.pone.0057622] [PMID] [PMCID].
[13] Filip R, Possemiers S, Heyerick A, Pinheiro I, Raszewski G, Davicco MJ, et al. Twelve-month consumption of a polyphenol extract from olive (Olea europaea) in a double blind, randomized trial increases serum total osteocalcin levels and improves serum lipid profiles in postmenopausal women with osteopenia. J Nutr Health Aging. 2015; 19(1):77-86. [DOI:10.1007/s12603-014-0480-x] [PMID].

[14] Abunab H, Dator WL, Hawamdeh S. Effect of olive leaf ex tract on glucose levels in diabetes-induced rats: A systematic review and meta-analysis. J Diabetes. 2017; 9(10):947-57. [DOI:10.1111/1753-0407.12508] [PMID].

[15] Susalit E, Agus N, Effendi I, Tjandrawinata RR, Nofiarny D, Perrinjaquet-Moccetti T, et al. Olive (Olea europaea) leaf extract effective in patients with stage-1 hypertension: comparison with Captopril. Phytomedicine. 2011; 18(4):251-8. [DOI:10.1016/j.phymed.2010.08.016] [PMID].

[16] Zhang Z, Joyce BT, Kresovich JK, Zheng $\mathrm{Y}$, Zhong J, Patel R, et al. Blood pressure and expression of microRNAs in whole blood. PloS One. 2017; 12(3):e0173550. [DOI:10.1371/journal.pone.0173550] [PMID] [PMCID].

[17] Karamali M, Eghbalpour S, Rajabi S, Jamilian M, Bahmani F, Tajabadi-Ebrahimi M, et al. Effects of probiotic supplementation on hormonal profiles, biomarkers of inflammation and oxidative stress in women with polycystic ovary syndrome: A randomized, double-blind, placebo-controlled trial. Arch Iran Med. 2018; 21(1):1-7. [In Persian]

[18] Benzie IF, Strain JJ. The ferric reducing ability of plasma (FRAP) as a measure of "antioxidant power": The FRAP assay. Anal Biochem. 1996; 239(1):70-6. [DOI:10.1006/abio.1996.0292] [PMID].

[19] Beutler E, Gelbart T. Plasma glutathione in health and in patients with malignant disease. J Lab and Clin Med. 1985; 105(5):581-4.

[20] Janero DR. Malondialdehyde and thiobarbituric acid-reactivity as diagnostic indices of lipid peroxidation and peroxidative tissue injury. Free Radic Biol and Med. 1990; 9(6):515-40. [DOI:10.1016/0891-5849(90)90131-2].

[21] Rukmini M, D'souza B, D'souza V. Superoxide dismutase and catalase activities and their correlation with malondialdehyde in schizophrenic patients. Indian J Clin Biochem. 2004; 19(2):114-8. [DOI:10.1007/BF02894268] [PMID] [PMCID]

[22] Mirhashemi SM, Najafi V, Raygan F, Asemi Z. The effects of coenzyme Q10 supplementation on cardiometabolic markers in overweight type 2 diabetic patients with stable myocardial infarction: A randomized, double-blind, placebo-controlled trial. ARYA Atheroscler. 2016; 12(4):158-65. [In Persian]

[23] Perrinjaquet-Moccetti T, Busjahn A, Schmidlin C, Schmidt A Bradl B, Aydogan C. Food supplementation with an olive (Olea europaea L.) leaf extract reduces blood pressure in borderline hypertensive monozygotic twins. Phytother Res. 2008; 22(9):1239-42. [DOI:10.1002/ptr.2455] [PMID].

[24] Lockyer S, Rowland I, Spencer JPE, Yaqoob P, Stonehouse W. Impact of phenolic-rich olive leaf extract on blood pressure, plasma lipids and inflammatory markers: A randomised controlled trial. Eur J Nutr. 2017; 56(4):1421-32. [DOI:10.1007/ s00394-016-1188-y] [PMID] [PMCID]. 
[25] Romero $M$, Toral $M$, Gómez-Guzmán $M$, Jiménez R, Galindo $P$, Sánchez $M$, et al. Antihypertensive effects of oleuropeinenriched olive leaf extract in spontaneously hypertensive rats. Food Funct. 2016; 7(1):584-93. [DOI:10.1039/C5FO01101A] [PMID].

[26] Sun W, Wang X, Hou C, Yang L, Li H, Guo J, et al. Oleuropein improves mitochondrial function to attenuate oxidative stress by activating the Nrf2 pathway in the hypothalamic paraventricular nucleus of spontaneously hypertensive rats. Neuropharmacol. 2017; 113:556-66. [DOI:10.1016/j.neuropharm.2016.11.010] [PMID].

[27] Khalili A, Nekooeian AA, Khosravi MB. Oleuropein improves glucose tolerance and lipid profile in rats with simultaneous renovascular hypertension and type 2 diabetes. J Asian Nat Prod Res. 2017; 19(10):1011-21. [In Persian] [DOI:10.1080/10 286020.2017.1307834] [PMID].

[28] Baghaiee B, Karimi P, Ebrahimi K. Effects of a 12-week aerobic exercise on markers of hypertension in men. J Cardiovasc Thorac Res. 2018; 10(3):162-8. [In Persian] [DOI:10.15171/ jcvtr.2018.26] [PMID] [PMCID].

[29] Efentakis P, Iliodromitis EK, Mikros E, Papachristodoulou A, Dagres N, Skaltsounis AL, et al. Effects of the olive tree leaf constituents on myocardial oxidative damage and atherosclerosis. Planta Med. 2015; 81(08):648-54. [DOI:10.1055/s-0035-1546017] [PMID].

[30] Lins PG, Pugine SMP, Scatolini AM, de Melo MP. In vitro antioxidant activity of olive leaf extract (Olea europaea L.) and its protective effect on oxidative damage in human erythrocytes. Heliyon. 2018; 4(9):e00805. [DOI:10.1016/j.heliyon.2018. e00805] [PMID] [PMCID].

[31] Kendall M, Batterham M, Obied H, Prenzler PD, Ryan D, Robards K. Zero effect of multiple dosage of olive leaf supplements on urinary biomarkers of oxidative stress in healthy humans. Nutrition. 2009; 25(3):270-80. [DOI:10.1016/j. nut.2008.08.008] [PMID]. 